\title{
Hom-algebra structures
}

\author{
Abdenacer MAKHLOUF ${ }^{a}$ and Sergei D. SILVESTROV ${ }^{b}$ \\ ${ }^{a}$ Université de Haute Alsace, Laboratoire de Mathématiques, Informatique et Applications, \\ 4 rue des Frères Lumière, F-68093 Mulhouse, France \\ E-mail: Abdenacer.Makhlouf@uha.fr \\ ${ }^{b}$ Centre for Mathematical Sciences, Lund University, Box 118, SE-221 00 Lund, Sweden \\ E-mail: sergei.silvestrov@math.lth.se
}

\begin{abstract}
Hom-algebra structures are given on linear spaces by multiplications twisted by linear maps. Hom-Lie algebras and general quasi-Hom-Lie and quasi-Lie algebras were introduced by Hartwig, Larsson and Silvestrov as algebras embracing Lie algebras, super and color Lie algebras and their quasi-deformations by twisted derivations. In this paper we introduce and study Hom-associative, Hom-Leibniz and Hom-Lie admissible algebraic structures generalizing associative, Leibniz and Lie admissible algebras. Also, we characterize flexible Hom-algebras and explain some connections and differences between Hom-Lie algebras and Santilli's isotopies of associative and Lie algebras.
\end{abstract}

2000 MSC: 17A30, 16Y99, 17A01, 17A20, 17D25.

Keywords: Hom-Lie algebra, Hom-Associative algebra, Hom-Leibniz algebra, Hom-Lie admissible algebra, flexible algebra, classification.

\section{Introduction}

In $[4,5,6]$, the class of quasi-Lie algebras and subclasses of quasi-Hom-Lie algebras and HomLie algebras had been introduced. These classes of algebras are tailored in a way suitable for simultaneous treatment of the Lie algebras, Lie superalgebras, color Lie algebras and deformations arising in connection with twisted, discretized or deformed derivatives and corresponding generalizations, discretizations and deformations of vector fields and differential calculus. It had been shown in $[4,5,6,7]$ that the class of quasi-Hom-Lie algebras contains as a subclass on one hand the color Lie algebras and in particular Lie superalgebras and Lie algebras, and on another hand various known and new single and multi-parameter families of algebras obtained using twisted derivations and constituting deformations and quasi-deformations of universal enveloping algebras of Lie and color Lie algebras and of algebras of vector fields. The main feature of quasi-Lie algebras, quasi-Hom-Lie algebras and Hom-Lie algebras is that the skew-symmetry and the Jacobi identity are twisted by several deforming twisting maps and also in quasi-Lie and quasi-Hom-Lie algebras the Jacobi identity in general contains 6 twisted triple bracket terms.

In this paper, we provide a different way for constructing Hom-Lie algebras by extending the fundamental construction of Lie algebras from associative algebras via commutator bracket multiplication. To this end we define the notion of Hom-associative algebras generalizing associative algebras to a situation where associativity law is twisted, and show that the commutator product defined using the multiplication in a Hom-associative algebra leads naturally to Hom-Lie algebras. We introduce also Hom-Lie admissible algebras and more general $G$-Hom-associative algebras with subclasses of Hom-Vinberg and pre-Hom-Lie algebras, generalizing to the twisted situation Lie admissible algebras, $G$-associative algebras, Vinberg and pre-Lie algebras respectively, and show that for these classes of algebras the operation of taking commutator leads to Hom-Lie algebras as well. We construct all the twistings so that the brackets

$$
\left[X_{1}, X_{2}\right]=2 X_{2}, \quad\left[X_{1}, X_{3}\right]=-2 X_{3}, \quad\left[X_{2}, X_{3}\right]=X_{1}
$$


determine a three-dimensional Hom-Lie algebra, generalizing $\mathfrak{s l}(2)$. Finally, we provide for a subclass of twistings, a list of all three-dimensional Hom-Lie algebras. For some values of structure constants, this list contains all three-dimensional Lie algebras. The families of HomLie algebras in these list can be viewed as deformations of Lie algebras into a class of Hom-Lie algebras.

The paper is organized as follows. In Section 1, we summarize the definitions of Homassociative, Hom-Leibniz algebra and Hom-Lie algebra. In Section 2, we extend the classical concept of Lie admissible algebra to Hom-Lie admissible algebra. In Section 3 we review and show the connections to Santilli's isotopies of associative and Lie algebras. In Section 4, we explore some general classes of Hom-Lie admissible algebras. For any subgroup $G$ of the permutation group $\mathcal{S}_{3}$, we introduce the $G$-Hom-associative algebras, show that they are Hom-Lie admissible and describe all these classes. Section 5 is devoted to some properties of flexible Hom-algebras. We extend the notion of flexibility to Hom-algebras and characterize flexible Hom-algebras using Jordan and Lie parts of the multiplication. In Section 6, we consider the algebraic varieties of finite-dimensional Hom-associative and Hom-Lie algebras. We also provide a characterization of all Hom-Lie algebras of $\mathfrak{s l}(2)$ type and construct all the 3-dimensional Hom-Lie algebras for a class of twisting homomorphisms.

\section{Hom-associative, Hom-Leibniz and Hom-Lie algebras}

Let $\mathbb{K}$ be an algebraically closed field of characteristic 0 and $V$ be a linear space over $\mathbb{K}$. Let $\alpha$ be a homomorphism of $V$. In the sequel we mean by Hom-algebra a triple $(V, \mu, \alpha)$ consisting of a linear space $V$, a bilinear map $\mu: V \times V \rightarrow V$ and a linear space homomorphism $\alpha: V \rightarrow V$.

Definition 1.1. A Hom-associative algebra is a triple $(V, \mu, \alpha)$ consisting of a linear space $V$, a bilinear map $\mu: V \times V \rightarrow V$ and a linear space homomorphism $\alpha: V \rightarrow V$ satisfying

$$
\mu(\alpha(x), \mu(y, z))=\mu(\mu(x, y), \alpha(z))
$$

A class of quasi-Leibniz algebras was introduced in [6] in connection to general quasi-Lie algebras following the standard Loday's conventions for Leibniz algebras (i.e. right Loday algebras). In the context of the subclass of Hom-Lie algebras one gets a class of Hom-Leibniz algebras.

Definition 1.2. A Hom-Leibniz algebra is a triple $(V,[\cdot, \cdot], \alpha)$ consisting of a linear space $V$, bilinear map $[\cdot, \cdot]: V \times V \rightarrow V$ and a linear space homomorphism $\alpha: V \rightarrow V$ satisfying

$$
[[x, y], \alpha(z)]=[[x, z], \alpha(y)]+[\alpha(x),[y, z]]
$$

In terms of the (right) adjoint homomorphisms $A d_{Y}: V \rightarrow V$ defined by $A d_{Y}(X)=[X, Y]$, the identity (1.1) can be written as

$$
A d_{\alpha(z)}([x, y])=\left[A d_{z}(x), \alpha(y)\right]+\left[\alpha(x), A d_{z}(y)\right]
$$

or in pure operator form

$$
A d_{\alpha(z)} \circ A d_{y}=A d_{\alpha(y)} \circ A d_{z}+A d_{A d_{z}(y)} \circ \alpha
$$

The Hom-Lie algebras were initially introduced by Hartwig, Larson and Silvestrov in [4] motivated initially by examples of deformed Lie algebras coming from twisted discretizations of vector fields. 
Definition 1.3 (Hartwig, Larsson, Silvestrov [4]). A Hom-Lie algebra is a triple $(V,[\cdot, \cdot], \alpha)$ consisting of a linear space $V$, bilinear map $[\cdot, \cdot]: V \times V \rightarrow V$ and a linear space homomorphism $\alpha: V \rightarrow V$ satisfying

$$
\begin{array}{ll}
{[x, y]=-[y, x]} & \text { (skew-symmetry) } \\
\circlearrowleft_{x, y, z}[\alpha(x),[y, z]]=0 & \text { (Hom-Jacobi identity) }
\end{array}
$$

for all $x, y, z$ from $V$, where $\circlearrowleft_{x, y, z}$ denotes summation over the cyclic permutation on $x, y, z$.

Using the skew-symmetry, one may write the Hom-Jacobi identity in the form (1.2). Thus, if a Hom-Leibniz algebra is skewsymmetric, then it is a Hom-Lie algebra.

Proposition 1.4. Every skew-symmetric bilinear map on a 2-dimensional linear space defines a Hom-Lie algebra.

Proof. The Hom-Jacobi identity (1.5) is satisfied for any triple $(x, x, y)$.

If we add the condition that the linear map $\alpha$ is an algebra homomorphism with respect to the bracket then we get restricted Hom-Lie algebras which are a special case of Quasi-Hom-Lie algebras. All these classes are a special case of the more general Quasi-Lie algebras [5, 6]. In the following, we recall the definition of Quasi-Lie algebras.

We denote by $\mathfrak{L}_{\mathbb{K}}(V)$ the set of linear maps of the linear space $V$ over the field $\mathbb{K}$.

Definition 1.5 (Larsson, Silvestrov [6]). A quasi-Lie algebra is a tuple $(V,[\cdot, \cdot], \alpha, \beta, \omega, \theta)$ consisting of

- $V$ is a linear space over $\mathbb{K}$,

- $[\cdot, \cdot]: V \times V \rightarrow V$ is a bilinear map that is called a product or bracket in $V$;

- $\alpha, \beta: V \rightarrow V$ are linear maps,

- $\omega: D_{\omega} \rightarrow \mathfrak{L}_{\mathbb{K}}(V)$ and $\theta: D_{\theta} \rightarrow \mathfrak{L}_{\mathbb{K}}(V)$ are maps with definition domains $D_{\omega}, D_{\theta} \subseteq V \times V$,

such that the following conditions hold:

- $(\omega$-symmetry) The product satisfies a generalized skew-symmetry condition

$$
[x, y]=\omega(x, y)[y, x], \quad \text { for all }(x, y) \in D_{\omega}
$$

- (quasi-Jacobi identity) The bracket satisfies a generalized Jacobi identity

$$
\circlearrowleft_{x, y, z}\{\theta(z, x)([\alpha(x),[y, z]]+\beta[x,[y, z]])\}=0
$$

for all $(z, x),(x, y),(y, z) \in D_{\theta}$.

Note that $[x, y]=\omega(x, y)[y, x]=\omega(x, y) \omega(y, x)[x, y]$ and hence $(\omega(x, y) \omega(y, x)-i d)[x, y]=0$, if $(x, y),(y, x) \in D_{\omega}$.

The class of Quasi-Lie algebras incorporates as special cases Hom-Lie algebras and more general quasi-Hom-Lie algebras (qhl-algebras) which appear naturally in the algebraic study of $\sigma$-derivations (see [4]) and related deformations of infinite-dimensional and finite-dimensional Lie algebras. To get the class of qhl-algebras one specifies $\theta=\omega$ and restricts attention to maps $\alpha$ and $\beta$ satisfying the twisting condition $[\alpha(x), \alpha(y)]=\beta \circ \alpha[x, y]$. Specifying this further by taking $D_{\omega}=V \times V, \beta=i d$ and $\omega=-i d$, one gets the class of Hom-Lie algebras including Lie algebras when $\alpha=i d$. The class of quasi-Lie algebras contains also color Lie algebras and in particular Lie superalgebras. 
Proposition 1.6. To any Hom-associative algebra $(V, \mu, \alpha)$, one may associate a Hom-Lie algebra defined for all $x, y \in V$ by the bracket $[x, y]=\mu(x, y)-\mu(y, x)$.

Proof. The bracket is obviously skewsymmetric and with a direct computation we have

$$
\begin{aligned}
{[\alpha(x),[y, z]] } & +[\alpha(z),[x, y]]+[\alpha(y),[z, x]]=\mu(\alpha(x), \mu(y, z))-\mu(\alpha(x), \mu(z, y)) \\
& -\mu(\mu(y, z), \alpha(x))+\mu(\mu(z, y), \alpha(x))+\mu(\alpha(z), \mu(x, y))-\mu(\alpha(z), \mu(y, x)) \\
& -\mu(\mu(x, y), \alpha(z))+\mu(\mu(y, x), \alpha(z))+\mu(\alpha(y), \mu(z, x))-\mu(\alpha(y), \mu(x, z)) \\
& -\mu(\mu(z, x), \alpha(y))+\mu(\mu(x, z), \alpha(y))=0
\end{aligned}
$$

\section{Hom-Lie-Admissible algebras}

The Lie admissible algebras were introduced by A. A. Albert in 1948. Physicists attempted to introduce this structure instead of Lie algebras. For instance, the validity of Lie-Admissible algebras for free particles is well known. These algebras arise also in classical quantum mechanics as a generalization of conventional mechanics (see [9], [10]). In this section, we extend to Homalgebras the classical concept of Lie-Admissible algebras.

Definition 2.1. Let $\mathcal{A}$ be a Hom-algebra structure on $V$ defined by the multiplication $\mu$ and a homomorphism $\alpha$. Then $\mathcal{A}$ is said to be Hom-Lie admissible algebra over $V$ if the bracket defined for all $x, y \in V$ by

$$
[x, y]=\mu(x, y)-\mu(y, x)
$$

satisfies the Hom-Jacobi identity $\circlearrowleft_{x, y, z}[\alpha(x),[y, z]]=0$ for all $x, y, z \in V$.

Remark 2.2. Since the commutator bracket (2.1) is always skew-symmetric, it makes any HomLie admissible algebra into a Hom-Lie algebra.

Remark 2.3. According to Proposition 1.6, any Hom-associative algebra is Hom-Lie admissible with the same twisting map.

It can be also checked that any Hom-Lie algebra with a twisting $\alpha$ is Hom-Lie admissible with the same twisting $\alpha$.

Proposition 2.4. Any Hom-Lie algebra $(V,[\cdot, \cdot], \alpha)$ is Hom-Lie admissible with the same twisting map $\alpha$.

Proof. For the commutator product $\langle x, y\rangle=[x, y]-[y, x]$ one has

$$
\begin{aligned}
\langle x, y\rangle & =[x, y]-[y, x]=-([y, x]-[x, y])=-\langle y, x\rangle \\
\circlearrowleft_{x, y, z}\langle\alpha(x),\langle y, z\rangle\rangle & =\circlearrowleft_{x, y, z}([\alpha(x),[y, z]]-[\alpha(x),[z, y]]-[[y, z], \alpha(x)]+[[z, y], \alpha(x)]) \\
& =4 \circlearrowleft_{x, y, z}[\alpha(x),[y, z]]=0
\end{aligned}
$$

For our further considerations it is convenient to introduce the following notation.

Definition 2.5. The $\alpha$-associator, associated to a product $\mu$ and a linear map $\alpha$, is a trilinear map $a_{\mu, \alpha}: V \times V \times V \rightarrow V$ defined by $a_{\mu, \alpha}\left(x_{1}, x_{2}, x_{3}\right)=\mu\left(\mu\left(x_{1}, x_{2}\right), \alpha\left(x_{3}\right)\right)-\mu\left(\alpha\left(x_{1}\right), \mu\left(x_{2}, x_{3}\right)\right)$.

In the following, we give a new characterization of Hom-Lie admissible algebras.

Let $\mathcal{A}=(V, \mu, \alpha)$ be a Hom-algebra and let $[x, y]=\mu(x, y)-\mu(y, x)$ be its commutator. We introduce the ternary map defined by $S(x, y, z):=a_{\mu, \alpha}(x, y, z)+a_{\mu, \alpha}(y, z, x)+a_{\mu, \alpha}(z, x, y)$. Then we have the following properties. 
Lemma 2.6. For all $x, y, z \in V$, the following equality holds

$$
S(x, y, z)=[\mu(x, y), \alpha(z)]+[\mu(y, z), \alpha(x)]+[\mu(z, x), \alpha(y)]
$$

Proof. The assertion follows by expanding commutators on the right hand side:

$$
\begin{aligned}
{[\mu(x, y), \alpha(z)] } & +[\mu(y, z), \alpha(x)]+[\mu(z, x), \alpha(y)]=\mu(\mu(x, y), \alpha(z))-\mu(\alpha(z), \mu(x, y)) \\
& +\mu(\mu(y, z), \alpha(x))-\mu(\alpha(x), \mu(y, z))+\mu(\mu(z, x), \alpha(y))-\mu(\alpha(y), \mu(z, x)) \\
& =S(x, y, z)
\end{aligned}
$$

Proposition 2.7. A Hom-algebra $\mathcal{A}$ is Hom-Lie admissible if and only if $S(x, y, z)=S(x, z, y)$ for all $x, y, z \in V$.

Proof. The assertion follows from

$$
\begin{aligned}
S(x, z, y)-S(x, y, z) & =[\mu(x, z), \alpha(y)]+[\mu(z, y), \alpha(x)]+[\mu(y, x), \alpha(z)] \\
& -[\mu(x, y), \alpha(z)]-[\mu(y, z), \alpha(x)]-[\mu(z, x), \alpha(y)] \\
& =\circlearrowleft_{x, y, z}[\alpha(x),[y, z]]
\end{aligned}
$$

\section{Lie-Santilli isotopies of associative and Lie algebras}

Already as early as 1967, Santilli considered in [11] the two-parametric deformations (mutations) of the Lie commutator bracket in an associative algebra, $(A, B)=p A B-q B A$, where $p$ and $q$ are scalar parameters and $A$ and $B$ are elements in the associative algebra (typically algebra of matrices or linear operators); and in 1978, Santilli $[12,13,14]$ extended it to "operatordeformations" of the Lie product as follows $(A, B)=A P B-B Q A$, where $P$ and $Q$ are fixed elements in the underlying associative algebra. The motivation for introducing non-associative generalizations of the bracket multiplication came from attempts to resolve certain limitations of conventional formalism of classical and quantum mechanics. Subsequently, in numerous works including articles and books by Santilli and other authors, the evolution equations based on such deformed brackets, physical applications and consequences of introducing such generalized models have been investigated. The deformations of the commutator bracket multiplication introduced by Santilli in investigations of foundations of classical and quantum mechanics and hadronic physics, have reappeared in many incarnations both in Mathematics and Physics. We refer the reader to $[2,12,13,14,15,16,17,18,19,20]$ for further discussion and references on the models based on introduction of such non-associative deformed commutator bracket multiplications instead of commutator (Lie) bracket multiplication, their bi-module type generalizations (genoalgebras) as well as for a review of relation with other appearances of so deformed commutator brackets in physics and mathematics, for example in contexts related to quantum algebras, quantum groups, Lie algebras and superalgebras, Jordan and other classes of algebras.

The relation between Hom-Lie admissible algebras and non-associative algebras with Santilli's deformed bracket products is certainly an interesting direction for further investigation. Here we would like to highlight some interrelations and differences between considered algebraic objects.

The Santilli's products with arbitrary $P$ and $Q$ are not anti-symmetric in general except when $P$ and $Q$ are specially interrelated within the underlying algebra, for example when $P=Q$ that is $(A, B)=A Q B-B Q A$. It can also be checked that the Lie-Santilli bracket product $(A, B)=A Q B-B Q A$ satisfies the Lie algebras Jacobi identity, which is not the case in general for $P \neq Q$. Thus the associative algebras with the modified product $A \times_{Q} B=A Q B$ are Lie admissible algebras. Since $\langle A, B\rangle=(A, B)-(B, A)=A(P+Q) B-B(P+Q) A$ the Santilli's deformed commutator product $(A, B)=A P B-B Q A$ defines a Lie admissible algebra as well. 
Remark 3.1. The Hom-Lie admissible algebras are Lie admissible algebras typically when the twisting mapping $\alpha$ built into their definition is the scalar multiple of the identity mapping. It could thus be of interest to describe, for a given twisting $\alpha$, as sharp as possible conditions on $P$ and $Q$ in order for the general Santilli's bracket product $(A, B)=A P B-B Q A$ to define a Hom-Lie algebra or more general Hom-Leibniz algebra with twisting $\alpha$.

Remark 3.2. It is possible also that Hom-Lie admissible algebras can be studied using Santilli's formalism of genoalgebras, the abstract bi-module type extension of the deformed commutator brackets $(A, B)=A P B-B Q A$, but after this formalism is appropriately modified to suit the Hom-Lie algebras context.

Santilli has considered also so called isotopies of associative and Lie algebras. Algebraic problem can be formulated as follows. Any associative algebra is Lie admissible since the commutator bracket on any associative algebra satisfies axioms of a Lie algebra. How can associative products in associative algebras be modified to yield as general as possible Lie admissible algebras? Can any Lie admissible algebra be obtained by such modifications from some associative algebra? Such modifications of associative and corresponding Lie algebras where called isotopies of associative and Lie algebras. In $[12,13,14,15,17,18,19]$, several general isotopies of associative products and associated Lie products have been identified. The most general of all presented there isotopies of a product for elements in an associative algebra $A$ over a field $\mathbb{K}$ is given by $x * y=a w x w T w y w$, where $a \in \mathbb{K}, w \in A, w^{2}=w \neq 0$, and $T$ is some extra element. The product $*$ is associative if $w^{2}=w$ and $T \in A$. Santilli allows $T$ to be some extra element outside $A$. Then a special care is needed on algebraic side in order to make involved objects and maps to be properly defined. If $a(w)(x)(w) T(w)(y)(w)$ is not identified with some element of $A$, then the new product $*$ is taking values in some generally non-associative algebra $A_{T}$ generated, as a linear space over $\mathbb{K}$, by elements $x \in A$ and formal expressions of the form $x_{1} T x_{2} T x_{3} \ldots x_{n-1} T x_{n} \in A T A T A \ldots A T A$ for $x_{1}, \ldots, x_{n} \in A$ for integers $n \geq 2$, whatever these expressions mean. With a $\mathbb{K}$-bilinear product on $A_{T}$ defined for $x, x_{1}, \ldots, x_{n}, y_{1}, \ldots, y_{m} \in A$ by

$$
\begin{aligned}
& \left(x_{1} T x_{2} T x_{3} \ldots x_{n-1} T x_{n}\right)\left(y_{1} T y_{2} T y_{3} \ldots y_{n-1} T y_{m}\right)= \\
& =x_{1} T x_{2} T x_{3} \ldots x_{n-1} T\left(x_{n} y_{1}\right) T y_{2} T y_{3} \ldots y_{m-1} T y_{m} \\
& x\left(x_{1} T x_{2} T x_{3} \ldots x_{n-1} T x_{n}\right)=\left(x x_{1}\right) T x_{2} T x_{3} \ldots x_{n-1} T x_{n} \\
& \left(x_{1} T x_{2} T x_{3} \ldots x_{n-1} T x_{n}\right) x=x_{1} T x_{2} T x_{3} \ldots x_{n-1} T\left(x_{n} x\right)
\end{aligned}
$$

in particular, the equalities $u(x T y)=(u x) T(y),(x T y) v=(x) T(y v), u(x T y) v=(u x) T(y v)$ hold for $u, x, y, v \in A$. Then the expression $x * y=a(w)(x)(w) T(w)(y)(w)$ yields again an element from $A_{T}$ for $x, y \in A_{T}$, and we get the product $*$ on the algebra $A_{T}$. If now $w^{2}=w$, then $*$ satisfies the associativity condition $x *(y * z)=(x * y) * z$ on $A_{T}$. Really, for

$$
x=x_{1} T x_{2} T x_{3} \ldots x_{n-1} T x_{n}, \quad y=y_{1} T y_{2} T y_{3} \ldots y_{n-1} T y_{m}, \quad z=z_{1} T z_{2} T z_{3} \ldots z_{n-1} T z_{k}
$$

using $w^{2}=w$, one gets

$$
\begin{aligned}
x *(y * z)= & a(w)\left(x_{1} T x_{2} T x_{3} \ldots x_{n-1} T x_{n}\right)(w) T(w) \\
& \left(a(w)\left(y_{1} T y_{2} T y_{3} \ldots y_{n-1} T y_{m}\right)(w) T(w)\left(z_{1} T z_{2} T z_{3} \ldots z_{n-1} T z_{k}\right)(w)\right)(w) \\
= & a^{2}(w)\left(x_{1} T x_{2} T x_{3} \ldots x_{n-1} T x_{n}\right)(w) T \\
& (w)\left(y_{1} T y_{2} T y_{3} \ldots y_{n-1} T y_{m}\right)(w) T(w)\left(z_{1} T z_{2} T z_{3} \ldots z_{n-1} T z_{k}\right)(w) \\
= & a(w)\left(a(w)\left(x_{1} T x_{2} T x_{3} \ldots x_{n-1} T x_{n}\right)(w) T(w)\right. \\
& \left.\left(y_{1} T y_{2} T y_{3} \ldots y_{n-1} T y_{m}\right)(w)\right)(w) T(w)\left(z_{1} T z_{2} T z_{3} \ldots z_{n-1} T z_{k}\right)(w) \\
= & (x * y) * z .
\end{aligned}
$$


One may show that this algebra carries a structure of Hom-associative algebra in the following way. Let $\alpha(s)=w s w$ for all $s \in A_{T}$. Then

$$
\begin{aligned}
\alpha(x) *(y * z) & =a w(w x w) w T w(a w y w T w z w) w=a w x w T w(a w y w T w z w) w \\
& =x *(y * z)=(x * y) * z \\
& =a w(a w x w T w y w) w T w z w=a w(\operatorname{awxwTwyw}) w T w(w z w) w \\
& =(x * y) * \alpha(z)
\end{aligned}
$$

The map $\alpha: s \mapsto w s w$ is a linear map on $A_{T}$, and $\left(A_{T}, *\right)$ is at the same time Hom-associative with non-trivial twisting map $\alpha$. However, since the product $*$ on $A_{T}$ is associative, the algebra $\left(A_{T}, *\right)$ is Lie admissible, that is a Hom-Lie admissible with the trivial twisting map $i d_{A_{T}}$.

If $w^{2} \neq w$, then the above reduction of Hom-associativity with the twisting map $\alpha: s \mapsto w s w$ to associativity is not working. Moreover, on many associative algebras $A$, there are linear maps $\alpha$, which can not be represented on the form $x \mapsto u x v$ at all. This explains why the classes of Hom-associative and Hom-Lie (admissible) algebras are different from Lie-Santilli algebras and isotopies of associative and Lie algebras. Whereas the relation between Hom-associative and Hom-Lie (admissible) algebras resembles that between associative and Lie algebras, the Hom-associative algebras are mostly not associative, and Hom-Lie (admissible) algebras are mostly not Lie (admissible) algebras. Only with special choices of $\alpha$ like above one recovers the associative and Lie algebra properties holding also in the case of Santilli's isotopies of associative and Lie algebras.

Remark 3.3. We assumed that $w \in A$ for simplicity of exposition. All conclusions above hold however even if $w$ as $T$ is an extra element possibly outside $A$, but of cause with $A_{T}$ replaced by a properly defined algebra $A_{T, w}$ built from elements $x \in A$ and the formal product expressions $x w, w x, x T, T x, T w, w T$, with product obeying the same rules as in $A_{T}$, allowing to group the elements in the products by brackets in the appropriate way, as if $w$ would belonged to $A$.

The other fundamental algebraic issue tackled by Santilli is imbedding of the scalar field into the algebras over this filed. If an algebra $A$ over the field $\mathbb{K}$ with a unit $1_{\mathbb{K}}$ has a unit $1_{A}$, then there is a canonical imbedding of the field into the algebra given by $i_{A}: c \mapsto c 1_{A}$ for $c \in \mathbb{K}$. Also one has $1_{A} x=x 1_{A}=x$. If the multiplication in $A_{T}$ is defined as $x * y=x T y$ (corresponding to $a=1_{\mathbb{K}}$ and $w=1_{A}$ ) then one still would like to have $1_{A_{T}} * x=x * 1_{A_{T}}=x$, which can be written as $1_{A_{T}} T x=x T 1_{A_{T}}=x$. Thus, if $T$ has left and right inverse $T^{-1}$, then $1_{A_{T}}=T^{-1}$. Also the canonical imbedding of the field into the new algebra yields $i_{A_{T}}\left(1_{\mathbb{K}}\right)=1_{\mathbb{K}} 1_{A_{T}}=1_{\mathbb{K}} T^{-1}$ and more generally $i_{A_{T}}(c)=c 1_{A_{T}}=c T^{-1}$ for $c \in \mathbb{K}$. These elements form a field inside $A_{T}$ with the unit $\hat{I}=1_{\mathbb{K}} T^{-1}$. This field $\hat{\mathbb{K}}$ is called isofield. Santilli noticed that dependence on $T$ of the new unit in the isofield, caused by the changed product in the algebra, is not just some complicating curiosity, but advantageous phenomena that opens new vast fundamental opportunities in physics, differential geometry, tensor calculus and beyond. This is because, while the unit $1_{\mathbb{K}}$ in the scalar field $\mathbb{K}$ is fixed, $T$ and thus the unit $\hat{I}$ in the isofield $\hat{\mathbb{K}}$ can be chosen to depend on the non-linear functionals or expressions in some other parameters, functions, their derivatives, integrals, etc., in physics having interpretation as time, position, speed, momentum, acceleration, mass, energy, etc. This dependence may be well highly nonlinear. Santilli has made an effort in systematic analysis of how the new algebra structures and introduction of isofield $\hat{\mathbb{K}}$ and parameter dependent isounits effect the equations of motion, time evolution and other basics of Hamiltonian and quantum mechanics. These first steps open a huge field for further research in many directions of interest in physics and mathematics. 


\section{Classification of Hom-Lie admissible algebras}

In the following, we explore some other general classes of Hom-Lie admissible algebras, $G$-Homassociative algebras, extending the class of Hom-associative algebras.

Definition 4.1. Let $G$ be a subgroup of the permutations group $\mathcal{S}_{3}$, a Hom-algebra on $V$ defined by the multiplication $\mu$ and a homomorphism $\alpha$ is said to be $G$-Hom-associative if

$$
\sum_{\sigma \in G}(-1)^{\varepsilon(\sigma)}\left(\mu\left(\mu\left(x_{\sigma(1)}, x_{\sigma(2)}\right), \alpha\left(x_{\sigma(3)}\right)\right)-\mu\left(\alpha\left(x_{\sigma(1)}\right), \mu\left(x_{\sigma(2)}, x_{\sigma(3)}\right)\right)=0\right.
$$

where $x_{i}$ are in $V$ and $(-1)^{\varepsilon(\sigma)}$ is the signature of the permutation $\sigma$.

The condition (4.1) may be written

$$
\sum_{\sigma \in G}(-1)^{\varepsilon(\sigma)} a_{\mu, \alpha} \circ \sigma=0
$$

where $\sigma\left(x_{1}, x_{2}, x_{3}\right)=\left(x_{\sigma(1)}, x_{\sigma(2)}, x_{\sigma(3)}\right)$.

Remark 4.2. If $\mu$ is the multiplication of a Hom-Lie admissible Lie algebra, then the condition (4.1) is equivalent to the property that the bracket defined by $[x, y]=\mu(x, y)-\mu(y, x)$ satisfies the Hom-Jacobi identity, or equivalently

$$
\sum_{\sigma \in \mathcal{S}_{3}}(-1)^{\varepsilon(\sigma)}\left(\mu\left(\mu\left(x_{\sigma(1)}, x_{\sigma(2)}\right), \alpha\left(x_{\sigma(3)}\right)\right)-\mu\left(\alpha\left(x_{\sigma(1)}\right), \mu\left(x_{\sigma(2)}, x_{\sigma(3)}\right)\right)\right)=0
$$

which may be written as

$$
\sum_{\sigma \in \mathcal{S}_{3}}(-1)^{\varepsilon(\sigma)} a_{\mu, \alpha} \circ \sigma=0
$$

Proposition 4.3. Let $G$ be a subgroup of the permutations group $\mathcal{S}_{3}$. Then any $G$-Homassociative algebra is a Hom-Lie admissible algebra.

Proof. The skewsymmetry follows straightaway from the definition.

We have a subgroup $G$ in $\mathcal{S}_{3}$. Take the set of conjugacy class $\{g G\}_{g \in I}$ where $I \subseteq G$, and for any $\sigma_{1}, \sigma_{2} \in I, \sigma_{1} \neq \sigma_{2} \Rightarrow \sigma_{1} G \bigcap \sigma_{1} G=\emptyset$. Then

$$
\sum_{\sigma \in \mathcal{S}_{3}}(-1)^{\varepsilon(\sigma)} a_{\mu, \alpha} \circ \sigma=\sum_{\sigma_{1} \in I} \sum_{\sigma_{2} \in \sigma_{1} G}(-1)^{\varepsilon\left(\sigma_{2}\right)} a_{\mu, \alpha} \circ \sigma_{2}=0
$$

The $G$-associative algebra in classical case was studied in [3]. The result may be extended to Hom-structures in the following way.

The subgroups of $\mathcal{S}_{3}$ are

$$
G_{1}=\{I d\}, \quad G_{2}=\left\{I d, \tau_{12}\right\}, \quad G_{3}=\left\{I d, \tau_{23}\right\}, \quad G_{4}=\left\{I d, \tau_{13}\right\}, \quad G_{5}=A_{3}, \quad G_{6}=\mathcal{S}_{3}
$$

where $A_{3}$ is the alternating group and where $\tau_{i j}$ is the transposition between $i$ and $j$.

We obtain the following type of Hom-Lie admissible algebras.

- The $G_{1}$-Hom-associative algebras are the Hom-associative algebras defined above.

- The $G_{2}$-Hom-associative algebras satisfy the condition

$$
\mu(\alpha(x), \mu(y, z))-\mu(\alpha(y), \mu(x, z))=\mu(\mu(x, y), \alpha(z))-\mu(\mu(y, x), \alpha(z))
$$

When $\alpha$ is the identity the algebra is called Vinberg algebra or left symmetric algebra. 
- The $G_{3}$-Hom-associative algebras satisfy the condition

$$
\mu(\alpha(x), \mu(y, z))-\mu(\alpha(x), \mu(z, y))=\mu(\mu(x, y), \alpha(z))-\mu(\mu(x, z), \alpha(y))
$$

When $\alpha$ is the identity the algebra is called pre-Lie algebra or right symmetric algebra.

- The $G_{4}$-Hom-associative algebras satisfy the condition

$$
\mu(\alpha(x), \mu(y, z))-\mu(\alpha(z), \mu(y, x))=\mu(\mu(x, y), \alpha(z))-\mu(\mu(z, y), \alpha(x))
$$

- The $G_{5}$-Hom-associative algebras satisfy the condition

$$
\begin{aligned}
\mu(\alpha(x), \mu(y, z))+\mu(\alpha(y), \mu(z, x)) & +\mu(\alpha(z), \mu(x, y))=\mu(\mu(x, y), \alpha(z)) \\
& +\mu(\mu(y, z), \alpha(x))+\mu(\mu(z, x), \alpha(y))
\end{aligned}
$$

If the product $\mu$ is skewsymmetric then the previous condition is exactly the Hom-Jacobi identity.

- The $G_{6}$-Hom-associative algebras are the Hom-Lie admissible algebras.

Then, one may set the following generalization of pre-Lie (and Vinberg) algebras.

Definition 4.4. A Hom-pre-Lie algebra is a triple $(V, \mu, \alpha)$ consisting of a linear space $V$, a bilinear map $\mu: V \times V \rightarrow V$ and a homomorphism $\alpha$ satisfying

$$
\mu(\alpha(x), \mu(y, z))-\mu(\alpha(x), \mu(z, y))=\mu(\mu(x, y), \alpha(z))-\mu(\mu(x, z), \alpha(y))
$$

Remark 4.5. A Hom-Vinberg algebra is the opposite algebra of a Hom-pre-Lie algebra.

\section{$5 \quad$ Flexible algebras}

The study of flexible Lie admissible algebras was initiated by Albert [1] and investigated by number of authors, Myung, Okubo, Laufer, Tomber and Santilli, see [8] and [15]. The aim of this section is to extend the notions and results about flexible algebras to Hom-structures.

Definition 5.1. A Hom-algebra $\mathcal{A}=(V, \mu, \alpha)$ is called flexible if for any $x, y$ in $V$

$$
\mu(\mu(x, y), \alpha(x))=\mu(\alpha(x), \mu(y, x)))
$$

Remark 5.2. Using the $\alpha$-associator $a_{\mu, \alpha}(x, y, z)=\mu(\mu(x, y), \alpha(z))-\mu(\alpha(x), \mu(y, z))$ the condition (5.1) may be written as

$$
a_{\mu, \alpha}(x, y, x)=0
$$

The $\alpha$-associator $a_{\mu, \alpha}$ is a useful tool in the study of Hom-Lie and Hom-Lie admissible algebras.

Lemma 5.3. Let $\mathcal{A}=(V, \mu, \alpha)$ be a Hom-algebra. The following assertions are equivalent

1. $\mathcal{A}$ is flexible.

2. For any $x, y$ in $V, a_{\mu, \alpha}(x, y, x)=0$.

3. For any $x, y, z$ in $V, a_{\mu, \alpha}(x, y, z)=-a_{\mu, \alpha}(z, y, x)$.

Proof. The equivalence of the first two assertions follows from the definition, and of last two assertions since $a_{\mu, \alpha}(x-z, y, x-z)=0$ holds by definition and is equivalent to $a_{\mu, \alpha}(x, y, z)+$ $a_{\mu, \alpha}(z, y, x)=0$ by linearity. 
Corollary 5.4. Any Hom-associative algebra is flexible.

Let $\mathcal{A}=(V, \mu, \alpha)$ be a Hom-algebra, where $\mu$ is the multiplication and $\alpha$ a homomorphism. We denote by $\mathcal{A}^{+}$the Hom-algebra over $V$ with a multiplication $x \bullet y=\frac{1}{2}(\mu(x, y)+\mu(y, x))$. We also denote by $\mathcal{A}^{-}$the Hom-algebra over $V$ where the multiplication is given by the commutator $[x, y]=\mu(x, y)-\mu(y, x)$.

Proposition 5.5. A Hom-algebra $\mathcal{A}=(V, \mu, \alpha)$ is flexible if and only if

$$
[\alpha(x), y \bullet z]=[x, y] \bullet \alpha(z)+\alpha(y) \bullet[x, z]
$$

Proof. Let $\mathcal{A}$ be a flexible Hom-algebra. By Lemma 5.3, this is equivalent to $a_{\mu, \alpha}(x, y, z)+$ $a_{\mu, \alpha}(z, y, x)=0$ for any $x, y, z$ in $V$, where $a_{\mu, \alpha}$ is the $\alpha$-associator of $\mathcal{A}$. This implies

$$
a_{\mu, \alpha}(x, y, z)+a_{\mu, \alpha}(z, y, x)+a_{\mu, \alpha}(x, z, y)+a_{\mu, \alpha}(y, z, x)-a_{\mu, \alpha}(y, x, z)-a_{\mu, \alpha}(z, x, y)=0
$$

By expansion, the previous relation is equivalent to $[\alpha(x), y \bullet z]=[x, y] \bullet \alpha(z)+\alpha(y) \bullet[x, z]$. Conversely, assume we have the condition (5.3) in Proposition. By setting $x=z$ in (5.4), one gets $a_{\mu, \alpha}(x, y, x)=0$. Therefore $\mathcal{A}$ is flexible.

\section{Algebraic varieties of Hom-structures}

Let $V$ be a $n$-dimensional $\mathbb{K}$-linear space and $\left\{e_{1}, \cdots, e_{n}\right\}$ be a basis of $V$. A Hom-algebra structure on $V$ with product $\mu$ is determined by $n^{3}$ structure constants $C_{i j}^{k}$, where $\mu\left(e_{i}, e_{j}\right)=$ $\sum_{k=0}^{n} C_{i j}^{k} e_{k}$ and by $\alpha$ which is given by $n^{2}$ structure constants $a_{i j}$, where $\alpha\left(e_{i}\right)=\sum_{j=0}^{n} a_{i j} e_{j}$.

If we require this algebra structure to be Hom-associative, then this limits the set of structure constants $\left(C_{i j}^{k}, a_{i j}\right)$ to a subvariety of $\mathbb{K}^{n^{3}+n^{2}}$ defined by the following polynomial equations:

$$
\sum_{l, m=1}^{n} a_{i l} C_{j k}^{m} C_{l m}^{s}-a_{k m} C_{i j}^{l} C_{l m}^{s}=0, \quad i, j, k, s=1, \ldots, n
$$

The algebraic variety of $n$-dimensional Hom-associative algebras is denoted by HomAss ${ }_{n}$. Note that the equations are given by cubic polynomials.

If we consider the $n$-dimensional unital Hom-associative algebras, then we obtain a subvariety (which we denote by $\mathrm{Hom} \mathrm{Alg}_{n}$ ) determined by the following polynomial equations:

$$
\begin{aligned}
& \sum_{l, m=1}^{n} a_{i l} C_{j k}^{m} C_{l m}^{s}-a_{k m} C_{i j}^{l} C_{l m}^{s}=0 \\
& C_{i 1}^{k}=C_{1 i}^{k}=\left\{\begin{array}{l}
1, \text { if } i=k \\
0, \text { if } i \neq k
\end{array} \quad \text { for } i, j, k, s=1, \ldots, n\right.
\end{aligned}
$$

If we require this algebra structure to be Hom-Lie, then the structure constants $\left\{\left(C_{i j}^{k}\right)_{i<j},\left(a_{i j}\right)\right\}$ determine a subvariety of $\mathbb{K}^{n^{2}(n+1) / 2}$ defined by the following system of polynomial equations

$$
\sum_{l, m=1}^{n} a_{i l} C_{j k}^{m} C_{l m}^{s}+a_{j l} C_{k i}^{m} C_{l m}^{s}+a_{k l} C_{i j}^{m} C_{l m}^{s}=0
$$

where $C_{i j}^{k}=-C_{j i}^{k}$.

The variety of $n$-dimensional Hom-Lie algebras is denoted by $\mathrm{HomLie}_{n}$.

One can do the same for every Hom-Lie admissible structure. If the algebras are parameterized by their structure constants and the structure constants of the homomorphism, the set of $n$-dimensional algebras of given structure carries a structure of algebraic varieties in $\mathbb{K}^{n^{3}+n^{2}}$ defined by a system of cubic polynomials. 
A point in such an algebraic variety (e.g. $\mathrm{HomAss}_{n}, \mathrm{HomLie}_{n}$ ) represents an $n$-dimensional algebra (e.g. Hom-associative, or Hom-Lie), along with a particular choice of basis. A change of basis may give rise to a (possibly) different point of the algebraic variety. The group $G L(n, \mathbb{K})$ acts on the algebraic varieties of Hom-structures by the so-called "transport of structure" action defined as follows.

Let $\mathcal{A}$ be an algebra (e.g. Hom-associative, or Hom-Lie) defined by multiplication $\mu$ and a linear map $\alpha$. Given $f \in G L(n, \mathbb{K})$, the action $f \cdot \mathcal{A}$ transports the structures by

$$
\begin{aligned}
f \cdot \mu(x, y) & =f^{-1} \mu(f(x), f(y)) \\
f \cdot \alpha(x) & =f^{-1} \alpha(f(x))
\end{aligned}
$$

The orbit of an algebra (e.g. Hom-associative, or Hom-Lie) $\mathcal{A}$ is given by

$$
\vartheta(\mathcal{A})=\left\{\mathcal{A}^{\prime}=f \cdot \mathcal{A}, f \in G L_{n}(\mathbb{K})\right\}
$$

The orbits are in 1-1-correspondence with the isomorphism classes of $n$-dimensional algebras.

\section{Three-dimensional Hom-Lie algebras}

In this section, we provide a characterization of all Hom-Lie algebras of $\mathfrak{s l}(2)$ type and construct all the 3-dimensional Hom-Lie algebras for a class of homomorphisms.

By a direct calculation, we obtain the following class of Hom-Lie algebras of $\mathfrak{s l}(2)$ type.

Proposition 7.1. Let $V$ be a three-dimensional $\mathbb{K}$-linear space and let $\left(X_{1}, X_{2}, X_{3}\right)$ be its basis. Any Hom-Lie algebra with the following brackets

$$
\left[X_{1}, X_{2}\right]=2 X_{2}, \quad\left[X_{1}, X_{3}\right]=-2 X_{3}, \quad\left[X_{2}, X_{3}\right]=X_{1}
$$

is given by homomorphism $\alpha$ defined, with respect to the previous basis, by a matrix of the form $\left(\begin{array}{ccc}a & d & c \\ 2 c & b & f \\ 2 d & \text { e } & b\end{array}\right)$ where $a, c, d, e, f$ are any parameters in $\mathbb{K}$.

This may be viewed as a quasi-deformation of $\mathfrak{s l}(2)$ in the class of Hom-Lie algebras. In the case where the matrix is the identity matrix one gets the classical Lie algebra $\mathfrak{s t}(2)$.

Remark 7.2. It would be interesting to compare this list with the quasi-Hom-Lie and Hom-Lie algebras of quasi-deformations of $\mathfrak{s l}(2)$ constructed in [7] using a quasi-Hom-Lie structure on twisted vector fields.

We provide in the following a list of all Hom-Lie algebras associated to the homomorphism given with respect to the basis $e_{1}, e_{2}, e_{3}$ of the 3 -dimensional $V$ by the following matrix $\left(\begin{array}{lll}a & 0 & 0 \\ 0 & b & 0 \\ 0 & 0 & b\end{array}\right)$. The list is obtained by solving the system of equations for the structure constants equivalent to axioms of Hom-Lie algebras. This list may be considered as a first partial step towards establishing a classification of 3 -dimensional hom-Lie algebras. In the list, $C_{i j}^{k}$ for $i, j, k=1,2,3$ are parameters in $\mathbb{K}$.

$$
\begin{aligned}
& {\left[e_{1}, e_{2}\right]_{1}=C_{12}^{1} e_{1}+C_{12}^{3} e_{3}} \\
& {\left[e_{1}, e_{3}\right]_{1}=C_{13}^{2} e_{2}} \\
& {\left[e_{2}, e_{3}\right]_{1}=C_{23}^{1} e_{1}+\frac{b C_{12}^{1}}{a} e_{3}} \\
& {\left[e_{1}, e_{2}\right]_{2}=\frac{a C_{23}^{3}}{b} e_{1}-C_{13}^{3} e_{2}+C_{12}^{3} e_{3}} \\
& {\left[e_{1}, e_{3}\right]_{2}=-\frac{a C_{23}^{2}}{b} e_{1}+C_{13}^{2} e_{2}+C_{13}^{3} e_{3}} \\
& {\left[e_{2}, e_{3}\right]_{2}=C_{23}^{1} e_{1}+C_{23}^{2} e_{2}+C_{23}^{3} e_{3}}
\end{aligned}
$$




$$
\begin{aligned}
& {\left[e_{1}, e_{2}\right]_{3}=0} \\
& {\left[e_{1}, e_{3}\right]_{3}=C_{13}^{1} e_{1}+C_{13}^{2} e_{2}} \\
& {\left[e_{2}, e_{3}\right]_{3}=C_{23}^{1} e_{1}} \\
& {\left[e_{1}, e_{2}\right]_{4}=C_{12}^{3} e_{3}} \\
& {\left[e_{1}, e_{3}\right]_{4}=C_{13}^{2} e_{2}+C_{13}^{3} e_{3}} \\
& {\left[e_{2}, e_{3}\right]_{4}=0} \\
& {\left[e_{1}, e_{2}\right]_{5}=C_{12}^{3} e_{3}} \\
& {\left[e_{1}, e_{3}\right]_{5}=C_{13}^{2} e_{2}} \\
& {\left[e_{2}, e_{3}\right]_{5}=C_{23}^{1} e_{1}} \\
& {\left[e_{1}, e_{2}\right]_{6}=C_{12}^{1} e_{1}+C_{12}^{3} e_{3}} \\
& {\left[e_{1}, e_{3}\right]_{6}=0} \\
& {\left[e_{2}, e_{3}\right]_{6}=C_{23}^{1} e_{1}} \\
& {\left[e_{1}, e_{2}\right]_{7}=C_{12}^{1} e_{1}+C_{12}^{2} e_{2}+C_{12}^{3} e_{3}} \\
& {\left[e_{1}, e_{3}\right]_{7}=0} \\
& {\left[e_{2}, e_{3}\right]_{7}=C_{23}^{1} e_{1}+C_{23}^{3} e_{3}} \\
& {\left[e_{1}, e_{2}\right]_{8}=-C_{13}^{3} e_{2}+C_{12}^{3} e_{3}} \\
& {\left[e_{1}, e_{3}\right]_{8}=C_{13}^{2} e_{2}+C_{13}^{3} e_{3}} \\
& {\left[e_{2}, e_{3}\right]_{8}=C_{23}^{1} e_{1}} \\
& {\left[e_{1}, e_{2}\right]_{9}=-C_{13}^{3} e_{2}+C_{12}^{3} e_{3}} \\
& {\left[e_{1}, e_{3}\right]_{9}=-\frac{a C_{23}^{2}}{b} e_{1}+C_{13}^{2} e_{2}+C_{13}^{3} e_{3}} \\
& {\left[e_{2}, e_{3}\right]_{9}=C_{23}^{1} e_{1}+C_{23}^{2} e_{2}} \\
& {\left[e_{1}, e_{2}\right]_{10}=0} \\
& {\left[e_{1}, e_{3}\right]_{10}=C_{13}^{1} e_{1}+C_{13}^{2} e_{2}+C_{13}^{3} e_{3}} \\
& {\left[e_{2}, e_{3}\right]_{10}=C_{23}^{1} e_{1}+C_{23}^{2} e_{2}} \\
& {\left[e_{1}, e_{2}\right]_{11}=C_{12}^{2} e_{2}+C_{12}^{3} e_{3}} \\
& {\left[e_{1}, e_{3}\right]_{11}=C_{13}^{2} e_{2}+C_{13}^{3} e_{3}} \\
& {\left[e_{2}, e_{3}\right]_{11}=0} \\
& {\left[e_{1}, e_{2}\right]_{12}=-\frac{C_{13}^{1} C_{23}^{3}}{C_{23}^{2}} e_{1}+C_{12}^{2} e_{2}-\frac{C_{13}^{3} C_{23}^{3}}{C_{23}^{2}} e_{3}} \\
& {\left[e_{1}, e_{3}\right]_{12}=C_{13}^{1} e_{1}-\frac{C_{12}^{2} C_{23}^{2}}{C_{23}^{3}} e_{2}+C_{13}^{3} e_{3}} \\
& {\left[e_{2}, e_{3}\right]_{12}=C_{23}^{1} e_{1}+C_{23}^{2} e_{2}+C_{23}^{3} e_{3}} \\
& {\left[e_{1}, e_{2}\right]_{13}=C_{12}^{3} e_{3}} \\
& {\left[e_{1}, e_{3}\right]_{13}=-\frac{a C_{23}^{2}}{b} e_{1}+C_{13}^{2} e_{2}} \\
& {\left[e_{2}, e_{3}\right]_{13}=C_{23}^{1} e_{1}+C_{23}^{2} e_{2}} \\
& {\left[e_{1}, e_{2}\right]_{14}=C_{12}^{1} e_{1}-C_{13}^{3} e_{2}+\frac{C_{12}^{1} C_{13}^{3}}{C_{13}^{1}} e_{3}} \\
& {\left[e_{1}, e_{3}\right]_{14}=C_{13}^{1} e_{1}-\frac{C_{13}^{1} C_{13}^{3}}{C_{12}^{1}} e_{2}+C_{13}^{3} e_{3}} \\
& {\left[e_{2}, e_{3}\right]_{14}=C_{23}^{1} e_{1}} \\
& {\left[e_{1}, e_{2}\right]_{15}=\frac{a C_{23}^{3}}{b} e_{1}+C_{12}^{2} e_{2}+\frac{C_{12}^{2} C_{23}^{3}}{C_{23}^{2}} e_{3}} \\
& {\left[e_{1}, e_{3}\right]_{15}=-\frac{\left((a-b) C_{12}^{2}-b C_{13}^{3}\right) C_{23}^{2}}{b C_{12}^{2}} e_{1}+C_{13}^{2} e_{2}+C_{13}^{3} e_{3}} \\
& {\left[e_{2}, e_{3}\right]_{15}=\frac{a C_{23}^{2} C_{23}^{3}}{b C_{12}^{2}} e_{1}+C_{23}^{2} e_{2}+C_{23}^{3} e_{3}} \\
& {\left[e_{1}, e_{2}\right]_{16}=\frac{a C_{23}^{3}}{b} e_{1}+\frac{C_{13}^{3} C_{23}^{3}}{C_{13}^{1}} e_{3}} \\
& {\left[e_{1}, e_{3}\right]_{16}=C_{13}^{1} e_{1}+C_{13}^{2} e_{2}+C_{13}^{3} e_{3}} \\
& {\left[e_{2}, e_{3}\right]_{16}=\frac{a C_{13}^{2} C_{23}^{3}}{b C_{13}^{3}} e_{1}+C_{23}^{3} e_{3}}
\end{aligned}
$$




$$
\begin{aligned}
& {\left[e_{1}, e_{2}\right]_{17}=-\frac{C_{13}^{1} C_{23}^{3}}{C_{23}^{2}} e_{1}-C_{13}^{3} e_{2}-\frac{C_{13}^{3} C_{23}^{3}}{C_{23}^{2}} e_{3}} \\
& {\left[e_{1}, e_{3}\right]_{17}=C_{13}^{3} e_{1}+\frac{C_{13}^{3} C_{23}^{2}}{C_{23}^{3}} e_{2}+C_{13}^{3} e_{3}} \\
& {\left[e_{2}, e_{3}\right]_{17}=C_{23}^{1} e_{1}+C_{23}^{2} e_{2}+C_{23}^{3} e_{3}} \\
& {\left[e_{1}, e_{2}\right]_{18}=C_{12}^{1} e_{1}+C_{12}^{2} e_{2}+C_{12}^{3} e_{3}} \\
& {\left[e_{1}, e_{3}\right]_{18}=-\frac{a C_{23}^{2}}{b} e_{1}-\frac{C_{12}^{2} C_{23}^{2}}{C_{12}^{1}} e_{2}} \\
& {\left[e_{2}, e_{3}\right]_{18}=\frac{a C_{12}^{1} C_{23}^{2}}{b C_{12}^{2}} e_{1}+C_{23}^{2} e_{2}} \\
& {\left[e_{1}, e_{2}\right]_{19}=-\frac{\left(b C_{12}^{2}+(b-a) C_{13}^{3}\right) C_{23}^{3}}{b C_{13}^{3}} e_{1}+C_{12}^{2} e_{2}+C_{12}^{3} e_{3}} \\
& {\left[e_{1}, e_{3}\right]_{19}=-\frac{a C_{23}^{2}}{b} e_{1}+\frac{C_{13}^{3} C_{23}^{2}}{C_{23}^{3}} e_{2}+C_{13}^{3} e_{3}} \\
& {\left[e_{2}, e_{3}\right]_{19}=\frac{a C_{23}^{2} C_{23}^{3}}{b C_{13}^{3}} e_{1}+C_{23}^{2} e_{2}+C_{23}^{3} e_{3}} \\
& {\left[e_{1}, e_{2}\right]_{20}=C_{12}^{2} e_{1}+C_{12}^{2} e_{2}+\frac{-a C_{13}^{3} C_{23}^{3}+b\left(C_{12}^{1} C_{13}^{1}+C_{12}^{2}+C_{13}^{3}\right) C_{23}^{3}}{b C_{13}^{1}+a C_{23}^{2}} e_{3}} \\
& {\left[e_{1}, e_{3}\right]_{20}=C_{13}^{1} e_{1}+\frac{b C_{12}^{2} C_{13}^{1}+a C_{12}^{2} C_{23}^{2}+b C_{12}^{2} C_{23}^{2}-b C_{13}^{3} C_{23}^{2}}{b C_{12}^{1}+a C_{23}^{3}} e_{2}+C_{13}^{3} e_{3}} \\
& {\left[e_{2}, e_{3}\right]_{20}=\frac{a C_{12}^{1} C_{23}^{2}+C_{13}^{1} C_{23}^{3}}{b\left(C_{12}^{2}+C_{13}^{3}\right)} e_{1}+C_{23}^{2} e_{2}+C_{23}^{3} e_{3}}
\end{aligned}
$$

\section{Acknowledgments}

This work was partially supported by the Crafoord foundation, The Swedish Foundation of International Cooperation in Research and Higher Education (STINT), The Royal Swedish Academy of Sciences, The Royal Physiographic Society in Lund, and the Liegrits network.

\section{References}

[1] A. A. Albert. Power associative rings, Trans. Amer. Math. Soc. 64 (1948), 552-597.

[2] R. M. Falcon-Ganfornina. J. Núnez Valdés, La isoteoria de Santilli. International Academic Press, Palm Harbor, FL, (2001).

[3] M. Goze and E. Remm. Lie-admissible algebras and operads. J. Algebra, 273 (2004), 129-152.

[4] J. T. Hartwig, D. Larsson and S. D. Silvestrov. Deformations of Lie algebras using $\sigma$-derivations. J. Algebra, 295 (2006), 314-361.

[5] D. Larsson and S. D. Silvestrov. Quasi-hom-Lie algebras, Central Extensions and 2-cocycle-like identities. J. Algebra, 288 (2005), 321-344.

[6] D. Larsson and S. D. Silvestrov. Quasi-Lie algebras. In "Noncommutative Geometry and Representation Theory in Mathematical Physics". J. Fuchs, J. Mickelsson, G. Rozenblioum, A. Stolin and A. Westenberg, Eds. Contemp. Math. 391 (2005), Amer. Math. Soc., Providence, RI, 241-248.

[7] D. Larsson, S. D. Silvestrov. Quasi-Deformations of $\mathfrak{s l}_{2}(\mathbb{F})$ using twisted derivations. Comm. in Algebra, 35 (2007), no. 12, 4303 - 4318.

[8] H. C. Myung. Lie-admissible Algebras. Hadronic J. 1 (1978), 169-193.

[9] H. C. Myung. Lie Algebras and Flexible Lie-admissible Algebras. Hadronic Press INC, Hadronic Press Monographs in Mathematics, 1, Massachusetts, 1982.

[10] H. C. Myung, S. Okubo and R. M. Santilli. Applications of Lie-admissible Algebras in Physics. Vol. I and II, Hadronic Press INC, Nonantum, Mass., 1978).

[11] R. M. Santilli. Imbedding of Lie algebras in nonassociative structures. Nuovo Cimento, A (10) 51 (1967), 570-576.

[12] R. M. Santilli. On a possible Lie-admissible covering of the Galilei relativity in Newtonian mechanics for nonconservative and Galilei form-noninvariant systems. Hadronic J. 1 (1978), 223-423.

[13] R. M. Santilli. Addendum to: "On a possible Lie-admissible covering of the Galilei relativity in Newtonian mechanics for nonconservative and Galilei form-noninvariant systems". Hadronic J. 1 (1978), 1279-1342. 
[14] R. M. Santilli. Need of subjecting to an experimental verification the validity within a hadron of Einstein's special relativity and Pauli's exclusion principle. Hadronic J. 1 (1978), 574-901.

[15] R. M. Santilli. Lie-admissible Approach to Hadronic Structure. Vol. I and II, Hadronic Press INC, Nonantum, Mass., 1979.

[16] R. M. Santilli. Foundations of Theoretical Mechanics, II. Birkhoffian Generalization of Hamiltonian Mechanics. Texts and Monographs in Physics. Springer-Verlag, New York-Berlin, 1983.

[17] R. M. Santilli. Invariant Lie-admissible formulation of quantum deformations. Found. Phys. 27 (1997), 1159-1177.

[18] R. M. Santilli. Origin, problematic aspects and invariant formulation of classical and operator deformations. Int. J. Modern Phys. A 14 (1999), 3157-3206.

[19] R. M. Santilli. Lie-admissible invariant treatment of irreversibility for matter and antimatter at the classical and operator levels. Nuovo Cimento, B (5) 121 (2006), 443-486.

[20] D. S. Sourlas and G. T. Tsagas. Mathematical Foundations of the Lie-Santilli Theory. "Naukova Dumka", Kiev; distributed by Hadronic Press, Inc., Palm Harbor, FL, 1993. 